\title{
Hip abductors and thigh muscles strength ratios and their relation to electromyography amplitude during split squat and walking lunge exercises
}

\author{
Petr Stastny ${ }^{1, *}$, James J. Tufano ${ }^{2}$, Michal Lehnert ${ }^{1}$, Artur Golas ${ }^{3}$, Amr Zaatar ${ }^{1}$, Zuzana Xaverova ${ }^{1}$, \\ and Adam Maszczyk ${ }^{3}$
}

${ }^{1}$ Faculty of Physical Culture, Palacký University Olomouc, Olomouc, Czech Republic; ${ }^{2}$ Centre for Exercise and Sports Science Research, Edith Cowan University, Joondalup, WA, Australia; and ${ }^{3}$ The Jerzy Kukuczka Academy of Physical Education in Katowice, Katowice, Poland

Copyright: (C) 2015 P. Stastny et al. This is an open access article licensed under the Creative Commons Attribution License (http://creativecommons.org/licenses/by/4.0/).

\begin{abstract}
Background: The hip abductors (HAB), quadriceps $(\mathrm{Q})$ and hamstrings $(\mathrm{H})$ reciprocal strength ratios are predictors of electromyography (EMG) amplitude during load carrying walking at moderate intensity. Therefore, these strength ratios might predict also the EMG during the exercises as walking lunge (WL) or split squat (SSq) at submaximal intensity. Objective: To determine whether the EMG amplitude of vastus mediali (VM), vastus laterali (VL), biceps femoris (BF) and gluteus medius (Gmed) is associated with muscle strength ratio during SSqs and WLs. To determine whether the EMG amplitude differs between individuals with $\mathrm{HAB} / \mathrm{H}$ ratio above and below one and between individuals with $\mathrm{H} / \mathrm{Q}$ or $\mathrm{HAB} / \mathrm{Q}$ ratio above and below 0.5 during SSqs and WLs. Methods: 17 resistance-trained men (age $29.6 \pm 4.6$ years) with at least 3 years of strength training performed in cross-sectional design $5 \mathrm{~s}$ maximal voluntary isometric contractions (MVIC) on an isokinetic dynamometer for knee extension, knee flexion, and hip abduction. The MVIC was used to normalize the EMG signal and estimate the individual strength ratios. Than participants performed WL and SSq for a 5 repetition maximum, to find out muscle activity at submaximal intensity of exercise. Results: The H/Q ratio was associated by Kendall's tau $(\tau)$ with $\mathrm{VM}(\tau=.33)$ and $\mathrm{BF}(\tau=-.71)$ amplitude, $\mathrm{HAB} / \mathrm{Q}$ ratio was associated with $\mathrm{BF}(\tau=-.43)$ and Gmed $(\tau=.38)$ amplitude, as well as $\mathrm{HAB} / \mathrm{H}$ was associated with VM $(\tau=-.41)$ and Gmed $(\tau=.74)$ amplitude. ANOVA results showed significant differences between SSq and $\mathrm{WL}\left(F(4,79)=10, p<.001, \eta_{\mathrm{p}}^{2}=.34\right)$ in Gmed amplitude, where WL resulted in higher Gmed amplitude compared to SSq. Other significant differences were found between $\mathrm{H} / \mathrm{Q}$ groups $\left(F(4,29)=3, p=.04, \eta_{\mathrm{p}}^{2}=.28\right)$ in VM and Gmed amplitude, where group with H/Q > 0.5 showed higher VMO amplitude and lower Gmed amplitude. Furthermore, significant difference was found for $\mathrm{HAB} / \mathrm{H}$ groups $\left(F(4,29)=4, p=.02, \eta_{\mathrm{p}}^{2}=.34\right)$ in $\mathrm{VM}$ amplitude, where group with $\mathrm{HAB} / \mathrm{H}<1$ showed higher VM amplitude. Conclusions: The ratios of $\mathrm{HAB}, \mathrm{H}$ and $\mathrm{Q}$ are able to predict Gmed, VM and BF activity during WL and SSq. WL resulted in higher activity level of Gmed than SSq, because WL includes the impact forces as part of lunge movement. WL should be used in resistance-training programme, if the strengthening of Gmed or VM is the aim.
\end{abstract}

Keywords: isokinetics, strength training, conditioning, muscle activity, strength exercise, gluteus medius, vastus medialis, vastus lateralis, hamstring, quadriceps

\section{Introduction}

Neuromuscular activity plays a key role in intramuscular and intermuscular coordination involved in joint centration. From this point of view, knee stability can be limited by muscle imbalance between vastus

\footnotetext{
* Address for correspondence: Petr Stastny, Department of Sport, Faculty of Physical Culture, Palacký University Olomouc, třída Míru 117, 77111 Olomouc, Czech Republic. E-mail: petr.stastny@upol.cz
}

medialis (VM), vastus lateralis (VL) (Bennell et al., 2010; Irish, Millward, Wride, Haas, \& Shum; Segal et al., 2010) and biceps femoris (BF) (Holcomb, Rubley, Lee, \& Guadagnoli, 2007; Kong \& Burns, 2010) during complex movements such as the Farmer's walk (Stastny et al., 2014) or walking lunges (Alkjær, Simonsen, Peter Magnusson, Aagaard, \& Dyhre-Poulsen, 2002). This instability could lead to pathologies, such as patellofemoral pain syndrome (Gilleard, McConnell, \& Parsons, 1998; Powers, Landel, \& Perry, 1996), 
and could be described by the activity ratio level as an appropriate muscle involvement with a hypothetical activity ratio of VM/VL approximately 1:1 (Irish et al., 2010). Another important stabilizer is gluteus medius (Gmed), which activity has been associated with both knee and hip instability (French, Dunleavy, \& Cusack, 2010; Kivlan \& Martin, 2012) as well as hip abductors (HAB) strength (Leetun, Ireland, Willson, Ballantyne, \& Davis, 2004).

Previous studies have found that the $\mathrm{HAB} / \mathrm{hip}$ adductor (HAD) strength ratio should be about 0.95 (Tyler, Nicholas, Campbell, \& McHugh, 2001), when a $\mathrm{HAB} / \mathrm{HAD}$ ratio of 0.78 was found in injured athletes. Other strength deficiencies might be found in conventional hamstring : quadriceps $(\mathrm{H} / \mathrm{Q})$ ratios of 0.5 to 0.6 (Aagaard, Simonsen, Magnusson, Larsson, \& DyhrePoulsen, 1998), which can increase along with tested speed up to the optimal ratio 1:1. The HAB, quadriceps $(\mathrm{Q})$ and hamstrings $(\mathrm{H})$ reciprocal strength ratio has been shown as possible predictor of electromyography (EMG) amplitude during load carrying walking (Stastny et al., 2015) at moderate intensity. The results of this study suggest that a $\mathrm{HAB} / \mathrm{H}$ ratio with a critical value of 1 influences the level of muscle bioelectrical activity of the Gmed and ratio of 0.5 might be used to separate the muscle activity by $\mathrm{H} / \mathrm{Q}$ or $\mathrm{HAB} / \mathrm{Q}$ ratios.

The imbalances in muscle activity or muscle strength are possible to reduce by exercises like forward walking lunge (WL), which is common therapeutic exercise, used in rehabilitation programs (Alkjær et al., 2002) or resistance-training programs (Jakobsen, Sundstrup, Andersen, Aagaard, \& Andersen, 2013) to developed lower limb strength. Some practitioners prefer the exercises without a landing phase such as split squats (SSq). WL display similar kinematics to stationary SSqs; the major difference between the two is that the dynamic nature of WL results in impact forces during landing whereas the SSqs do not since both feet are constantly fixed to the ground with one foot in front of the other. For practitioners use, it would be beneficial to know, whether WL or SSq produce higher muscle activity acting on knee and hip joint stability and if the strength ratio influences such involvement.

Therefore, purpose of this study is to determine if $\mathrm{H} / \mathrm{Q}, \mathrm{HAB} / \mathrm{H}$ and $\mathrm{HAB} / \mathrm{Q}$ strength ratios could predict muscle activation during SSqs and WLs. Specifically, this study has three objectives - to determine whether the EMG amplitude of the selected muscles is associated with muscle strength ratio during SSqs and WLs, to determine whether the EMG amplitude differs between individuals with $\mathrm{HAB} / \mathrm{H}$ ratio above and below 1 during SSqs and WLs and to determine whether the EMG amplitude differs between individuals with $\mathrm{H} / \mathrm{Q}$ or $\mathrm{HAB} / \mathrm{Q}$ ratio above and below 0.5 during $\mathrm{SSqs}$ and
WLs. The findings of the present study can be used for the optimization of exercise selection and for detailed evaluation of the strength deficit of individual muscles.

\section{Methods}

\section{Experimental approach to the problem}

The present investigation was a cross-sectional study and was performed in the biomechanics laboratory at the Faculty of Physical Culture, Palacký University Olomouc in May and June 2014. First, anthropometry measurements were taken to register participant height, body mass, leg length, knee width, ankle width, and greater trochanter to anterior superior iliac spine distance. The warm-up procedure consisted of $5 \mathrm{~min}$ of stationary cycling and one set of 25 bodyweight squats using different foot positions. After the warm up, EMG electrodes were secured on the skin over the belly of the VM, VL, RF, and Gmed and remained in the same place throughout the entire period of measurement. Then, participants performed a $5 \mathrm{~s}$ maximal voluntary isometric contraction on an isokinetic dynamometer for knee extension, knee flexion, and hip abduction to establish the EMG signal during maximum effort and isometric strength. 3D reflective markers were taped bilaterally on each subject before the WL and SSq exercises. Each exercise was performed first with bodyweight for 5 repetitions with one leg being the stance leg (the front leg while performing the lunge or squat) and 5 bodyweight repetitions with the other leg as the stance leg. Following a 1-3 min rest period, the first dumbbell load of $12.5 \mathrm{~kg}$ was used for the next set of 5 repetitions followed by another 1-3 min rest period and another increase of weight by approximately $12.5 \mathrm{~kg}$ for 5 repetitions. This process was repeated until the dumbbell mass exceeded the subject's ability to perform 5 repetitions (5RM). The recommendations of the American Society of Exercise Physiology were followed for this task (Brown \& Weir, 2001), so at least $60 \mathrm{~s}$ and a maximum of $5 \mathrm{~min}$ of rest were included between subsequent sets of each exercise (i.e. an increase in dumbbell load), but only the 5RM sets were included in the statistical analyses. Once the 5RM was determined for the first exercise, the same protocol was carried out for the remaining exercise in random order.

\section{Subjects}

The participants included 17 resistant trained males (age $29.6 \pm 4.6$ years, body mass $82.6 \pm 8.9 \mathrm{~kg}$ ) with at least 3 years of strength training experience in a selfreported structured training program, which included at least 2 resistance-training sessions for the lower 
limbs per week. At the time of data collection, none of the subjects had reported recent implementation of ipsilateral or contralateral loading of $\mathrm{SSq}$ or WL in their training programs. All participants were more than eighteen years old and lacked any pathologies or injuries. Written informed consent was provided by all participants, and the testing protocol was approved by the Ethics Committee at the Faculty of Physical Culture, Palacký University Olomouc in accordance with the ethical standards of the Helsinki Declaration of 1983. All participants were informed of and shown the testing protocols and all aspects of the study when they signed the written informed consent form for the study.

For statistical analysis, the participants' were divided into groups based on their $\mathrm{H} / \mathrm{Q}, \mathrm{HAB} / \mathrm{H}$ and $\mathrm{HAB} / \mathrm{Q}$ strength ratios (Table 1). The groups formed were participants with the results of $\mathrm{H} / \mathrm{Q} \geq 0.5$ (H/Q 1), H/Q < 0.5 (H/Q 2), HAB/H $\geq 1$ (HAB/H 1), $\mathrm{HAB} / \mathrm{H}<1 \quad(\mathrm{HAB} / \mathrm{H} 2), \mathrm{HAB} / \mathrm{Q} \geq 0.5(\mathrm{HAB} / \mathrm{Q} 1)$ and $\mathrm{HAB} / \mathrm{Q}<0.5(\mathrm{HAB} / \mathrm{Q} 2)$.

\section{Instrumentation}

Maximal voluntary isometric contraction (MVIC) was performed using an isokinetic dynamometer IsoMed 2000 (D \& R Ferstl, Hemau, Germany), which has been reported to have high reproducibility in peak torque measurement (Dirnberger, Kösters, \& Müller, 2012). The electromyography data were collected with a Noraxon 1400A device (Noraxon, Scottsdale, AZ, USA). Kinematic data were collected using a six-camera Vicon MX infra-red motion analysis system (Oxford Metrics, Oxford, UK) with find out validity (Windolf, Götzen, \& Morlock, 2008), which was completed using two force plates Kistler 9286AA (Kistler Instrumente, Winterthur, Switzerland). The Vicon motion analysis system, EMG, and force plate outputs were connected to and fully synchronized by analogue signal within the Vicon Nexus software (Oxford Metrics, Oxford, UK). These procedures are further explained below.

\section{Exercises}

Exercises were performed with both the dominant and non-dominant leg in randomized order.

The WLs started with the subjects standing with their feet together on one force platform and hands parallel to the trunk. The dumbbell was carried in one hand, and the lunge step was initiated by the contralateral leg stepping on the second force platform. The end of the exercise was defined as the end of foot contact with the second force platform, when returning the stance (loaded) leg back to starting position. The full range of the lunge was performed while keeping the trunk in an upright position, with the instruction "lunge down as far as possible" (Dwyer, Boudreau, Mattacola, Uhl, \& Lattermann, 2010).

For the SSqs, the participant started standing in the lunge position (described above) with one foot on each force plate with the supported (rear) leg standing on the toes and the stance leg flat on the force plate. The dumbbell was carried in one hand, which was contralateral to the stance leg. The full range of the SSq was performed while keeping the trunk in an upright position. The step distance was equal to the leg length, as determined by measuring from the anteriorsuperior iliac spine to the medial malleolus of the tibia (Boudreau et al., 2009; Dwyer et al., 2010) with the instruction "squat down as far as possible".

\section{Isometric strength measurement}

To obtain a maximal value of the EMG signal and isometric strength, subjects performed a $5 \mathrm{~s}$ maximal voluntary isometric contraction (MVIC) on the

Table 1

Isometric strength and strength ratio (Mean $\pm S D)$ in selected groups

\begin{tabular}{lcccc}
\hline Group & $\begin{array}{c}\text { Knee flexion } 75^{\circ} \\
\left(\mathrm{N} \cdot \mathrm{m}^{-1}\right)\end{array}$ & $\begin{array}{c}\text { Knee extension } 75^{\circ} \\
\left(\mathrm{N} \cdot \mathrm{m}^{-1}\right)\end{array}$ & $\begin{array}{c}\text { Hip abduction } 15^{\circ} \\
\left(\mathrm{N} \cdot \mathrm{m}^{-1}\right)\end{array}$ & Ratio $^{\mathrm{a}}$ \\
\hline H/Q 1 $(n=12)$ & $146 \pm 13$ & $246 \pm 63$ & $153 \pm 18$ & $0.64 \pm 0.18$ \\
H/Q 2 $(n=22)$ & $140 \pm 20$ & $341 \pm 47$ & $168 \pm 33$ & $0.41 \pm 0.04$ \\
HAB/Q 1 $(n=18)$ & $152 \pm 21$ & $276 \pm 35$ & $144 \pm 29$ & $0.63 \pm 0.16$ \\
HAB/Q 2 $(n=16)$ & $143 \pm 19$ & $331 \pm 37$ & $151 \pm 17$ & $0.46 \pm 0.04$ \\
HAB/H 1 $(n=18)$ & $139 \pm 17$ & $312 \pm 82$ & $181 \pm 28$ & $1.31 \pm 0.15$ \\
HAB/H 2 $(n=16)$ & $147 \pm 18$ & $301 \pm 52$ & $142 \pm 13$ & $0.98 \pm 0.05$ \\
\hline
\end{tabular}

Note. $\mathrm{H} / \mathrm{Q} 1$ = hamstring/quadriceps group 1, H/Q 2 = hamstring/quadriceps group 2, HAB/Q 1 = hip abductor/quadriceps group 1, HAB/Q 2 = hip abductor/quadriceps group 2, HAB/H 1 = hip abduc-

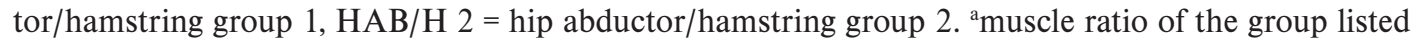
in the first column. 
dynamometer for unilateral knee flexion and extension and hip abduction on both legs. Each participant performed two consecutive measurements of each muscle group with $45 \mathrm{~s}$ rest intervals. A full passive range of motion and two submaximal isometric trials again resistance were performed on the dynamometer before executing each MVIC attempt to avoid injury.

First, the knee extensors (VM, VL) and knee flexors (BF) were tested for each leg. MVICs were measured in the standard sitting position with $75^{\circ} \mathrm{knee}$ flexion. The backrest of the dynamometer seat was set to an angle of $75^{\circ}$, and the angle of the hip joint was $100^{\circ}$. The arm of the dynamometer lever was fixed to the distal part of the shin, and the lower edge of the shin pad was placed $2.5 \mathrm{~cm}$ over the medial apex malleolus. Subjects were secured with belts in the pelvic region and the thigh region of the tested lower limbs, but did not interfere with the electrodes placed on the VM and VL. Adjustable straps and pads were placed on the shoulders, and participants held hand grips along the seats. The mechanical axis of the dynamometer was aligned with the knee axis according to the standard position for knee flexion/extension (Dirnberger et al., 2012).

Reference Gmed values for MVIC were obtained during side lying hip abduction (Burnet \& Pidcoe, 2009; Leetun et al., 2004). The subjects were positioned with the tested lower extremity in $10^{\circ}$ of hip abduction and $10^{\circ}$ of hip flexion. The arm of the dynamometer lever was fixed to lateral thigh of tested limb, $1 \mathrm{~cm}$ above the patella. To maintain the fixed testing position of the tested leg, a strap was used. The axis of the rotation of the dynamometer was aligned with the greater trochanter on the femur.

The greatest peak torque was used for statistical analyses and EMG value of that trial was used to normalize the EMG for \% MVIC. Participants were provided with concurrent visual feedback in the form of a strength curve displayed on the dynamometer monitor. Verbal encouragement was also provided.

\section{EMG measurement}

Raw EMG signals were recorded bilaterally by eight leads and sampled at $1000 \mathrm{~Hz}$. Two bipolar surface electrodes (adhesive disposable electrode - Kendall) were taped on each muscle with a $10 \mathrm{~mm}$ interelectrode distance. The input impedance was greater than $10 \mathrm{M} \Omega$ at $100 \mathrm{~Hz}$, with a frequency bandwidth of $16-800 \mathrm{~Hz}$ and a common mode rejection ratio of $60 \mathrm{~Hz}(80 \mathrm{~dB})$.

The electrodes for the VM were placed over the distal third of the muscle belly and were oriented $55^{\circ}$ to the vertical. The electrode for the VL was placed over the muscle belly in the distal third, and it was oriented $15^{\circ}$ to the vertical (Gilleard et al., 1998). The Gmed was located by palpating the iliac crest and placing electrodes parallel to the muscle fibres at $33 \%$ of the distance between the iliac crest and the greater trochanter (Bolgla \& Uhl, 2005, 2007), which is similar to the locations used by O'Sullivan, Smith, and Sainsbury (2010) for the posterior Gmed. The electrodes for the $\mathrm{BF}$ were placed over the distal third of the long head muscle belly. The ground electrode was placed over the tibia bone.

\section{D kinematics measurement}

Six cameras were spaced around the walking track with two force plates in the middle while the kinematic data were recorded at $200 \mathrm{~Hz}$ in accordance with the plug-in gait model (Davis, Õnpuu, Tyburski, \& Gage, 1991). Reflective markers that were $14 \mathrm{~mm}$ in diameter were attached bilaterally to the subject's skin over the following areas: the anterior superior iliac spine, posterior superior iliac spine, lateral thigh, lateral femoral epicondyle, tibia, lateral malleolus, heel, and metatarsal's head of the second toe. Force plates were used to detect and standardize the beginning of the foot contact during WLs with a contact sensitivity of $20 \mathrm{~N}$.

\section{Data acquisition}

The Vicon Nexus software program was used to compute knee angles in the sagittal plane and hip angles in the sagittal, frontal, and transverse planes. Kinematic values expressed in degrees were the range of motion (ROM) of the knee and hip during the whole exercise movement. These included hip abduction/adduction ROM, hip external/internal rotation ROM, hip flexion/extension ROM and knee flexion/extension ROM. ROM was calculated as an absolute difference in both directions of the selected movement. For example, knee flexion was measured from minimum to maximum flexion angles whereas hip external rotation may not have started from a neutral position, meaning that both internal and external rotation needed to be considered when calculating the total hip rotation ROM. All of these variables were obtained from both legs, but were only evaluated for the stance leg during the SSq and WL exercises.

EMG data were band pass filtered $(16-500 \mathrm{~Hz})$ and smoothed using a root mean square algorithm with sliding window function with a time constant of $25 \mathrm{~ms}$ and normalized to the EMG during MVIC. EMG mean amplitudes (expressed as \% MVIC) were used for statistical analyses as muscle activity values for Gmed, VM, VL and BF.

\section{Statistics}

All statistical analyses were performed in STATISTICA (Version 12; StatSoft, Tulsa, OK, USA) with 
$\alpha=.05$. The middle 3 repetitions of each leg during the $5 \mathrm{RM}$ trial of each exercise were averaged for further statistical analyses. The intraclass correlation coefficient (ICC) across 3 repetitions for each individual was determined to confirm whether the EMG and 3D measurements were stable within a subject. The Shapiro-Wilk test was performed to find out data normality of EMG in selected groups.

The Kendall's rank-order correlations (Kendall's tau " $\tau$ ") were used to determine the dependence between EMG amplitudes and strength ratio during both exercises without recognizing groups. Kendall's $\tau$ was used because this coefficient does not require any assumptions for correlation linearity, and it is not dependent on the number of involved cases (Sheskin, 2003).

The $2 \times 4$ (exercise $\times$ muscle) repeated measure analysis of variance (ANOVA) was used to compare differences in EMG between both exercises. Dependent variables were exercises without recognizing strength ratio. To determine whether EMG amplitude varied between each two separated groups, a $2 \times 4$ (group $\times$ muscle) ANOVA for repeated measures variance on four variables (muscles) was performed. Between subject (group) factors was used as a result. Both ANOVA analyses were followed by Tukey's post hoc tests. The effect size (partial eta squared $-\eta_{\mathrm{p}}^{2}$ ) of each test was calculated for all analyses and was classified according to Larson-Hall (2009), where $\eta_{\mathrm{p}}^{2:} .01$, $.06,0.14$ were estimated for small, moderate, large effect respectively. Statistical significance was set at $p<.05$.

\section{Results}

The within subject reliability showed the ICC values for EMG between .61 and .92 (Table 2), which is considered to be a moderate or high level of reliability (Chandler \& Brown, 2008).

The H/Q ratio was associated with $\mathrm{VM}(\tau=.33$, $p=.006)$ and $\mathrm{BF}(\tau=-.71, p<.001)$ amplitude, $\mathrm{HAB} / \mathrm{Q}$ ratio was associated with $\mathrm{BF}(\tau=-0.43, p<.001)$ and Gmed $(\tau=.38, p=.002)$ amplitude, as well as $\mathrm{HAB} / \mathrm{H}$ was associated with VM $(\tau=-.41, p<.001)$ and Gmed $(\tau=.74, p<.001)$ amplitude.

ANOVA results showed significant differences between SSq and WL $\left(F(4,79)=10, p<.001, \eta_{\mathrm{p}}^{2}=.34\right.$, power $\alpha=.98)$ in Gmed amplitude, where WL resulted in higher Gmed amplitude compare to SSq (Table 3 and Figure 1). Another significant differences were found between $\mathrm{H} / \mathrm{Q}$ groups $(F(4,29)=3, p=.04$, $\eta_{\mathrm{p}}^{2}=.28$, power $\alpha=.70$ ) in VM and Gmed amplitude, where H/Q 1 group showed higher VM amplitude and lower Gmed amplitude (Table 3 and Figure 2). Furthermore, significant difference was found for $\mathrm{HAB} / \mathrm{H}$ groups $\left(F(4,29)=4, p=.02, \eta_{\mathrm{p}}^{2}=.34\right.$, power $\left.\alpha=.82\right)$ in $\mathrm{VM}$ amplitude, where $\mathrm{HAB} / \mathrm{H} 2$ showed higher $\mathrm{VM}$ amplitude (Table 3 and Figure 2). No other differences among groups or exercises were found for any of the other variables.

\section{Discussion}

The purpose of this study was to determine if $\mathrm{H} / \mathrm{Q}$, $\mathrm{HAB} / \mathrm{H}$ or $\mathrm{HAB} / \mathrm{Q}$ strength ratios could predict the

Table 2

Normality by Shapiro-Wilk test in groups and within-subject reliability for EMG

\begin{tabular}{|c|c|c|c|c|c|c|c|c|}
\hline & \multicolumn{2}{|c|}{$\mathrm{VM}$} & \multicolumn{2}{|c|}{ VL } & \multicolumn{2}{|c|}{$\mathrm{BF}$} & \multicolumn{2}{|c|}{ Gmed } \\
\hline & WL & $\mathrm{SSq}$ & WL & $\mathrm{SSq}$ & WL & $\mathrm{SSq}$ & WL & $\mathrm{SSq}$ \\
\hline $\mathrm{H} / \mathrm{Q} 1(n=12)$ & .93 & .78 & .94 & .94 & .90 & .76 & .87 & .87 \\
\hline $\mathrm{H} / \mathrm{Q} 2(n=22)$ & .93 & .97 & .97 & .98 & .80 & .90 & .97 & .75 \\
\hline HAB/Q $1(n=18)$ & .94 & .95 & .97 & .95 & .78 & .87 & .91 & .92 \\
\hline $\mathrm{HAB} / \mathrm{Q} 2(n=16)$ & .82 & .85 & .90 & .92 & .91 & .92 & .93 & .75 \\
\hline $\mathrm{HAB} / \mathrm{H} 1(n=18)$ & .92 & .95 & .98 & .96 & .92 & .83 & .94 & .90 \\
\hline $\mathrm{HAB} / \mathrm{H} 2(n=16)$ & .90 & .85 & .94 & .92 & .74 & .88 & .91 & .77 \\
\hline $\mathrm{ICC}^{\mathrm{a}}$ & .84 & .85 & .82 & .86 & .61 & .75 & .89 & .92 \\
\hline$S E M^{\mathrm{a}}$ & 4.06 & 5.63 & 4.06 & 5.14 & 3.44 & 3.05 & 5.44 & 1.32 \\
\hline
\end{tabular}

Note. $\mathrm{VM}=$ vastus medialis, $\mathrm{VL}=$ vastus lateralis, $\mathrm{BF}=$ biceps femoris, $\mathrm{Gmed}=$ gluteus medius, $\mathrm{WL}=$ walking lunge, $\mathrm{SSq}=$ split squat, H/Q 1 = hamstring/quadriceps group 1, H/Q 2 = hamstring/quadriceps group 2, HAB/Q 1 = hip abductor/quadriceps group $1, \mathrm{HAB} / \mathrm{Q} 2$ = hip abductor/quadriceps group 2, $\mathrm{HAB} / \mathrm{H} 1=$ hip abductor/hamstring group 1, HAB/H 2 = hip abductor/hamstring group 2, ICC = intraclass correlation coefficient, SEM= standard error of measurement. ${ }^{a}$ without recognizing groups, statistical values were calculated for percentage of maximal voluntary isometric contraction. 
Table 3

EMG results by groups expressed as means and standard deviations

\begin{tabular}{|c|c|c|c|c|c|c|c|c|}
\hline & \multicolumn{2}{|c|}{ VM (\%MVIC) } & \multicolumn{2}{|c|}{ VL (\%MVIC) } & \multicolumn{2}{|c|}{ BF (\%MVIC) } & \multicolumn{2}{|c|}{ Gmed (\%MVIC) } \\
\hline & WL & $\mathrm{SSq}$ & WL & $\mathrm{SSq}$ & WL & $\mathrm{SSq}$ & WL & $\mathrm{SSq}$ \\
\hline All participants $(N=34)$ & $49 \pm 18$ & $40 \pm 18$ & $42 \pm 15$ & $38 \pm 14$ & $27 \pm 18$ & $19 \pm 13$ & $54 \pm 29 \dagger$ & $25 \pm 19 \dagger$ \\
\hline H/Q $1(n=12)$ & $59 \pm 23^{*}$ & $43 \pm 19$ & $39 \pm 14$ & $35 \pm 9$ & $24 \pm 11$ & $17 \pm 13$ & $40 \pm 23^{*}$ & $23 \pm 16$ \\
\hline $\mathrm{H} / \mathrm{Q} 2(n=22)$ & $45 \pm 12 *$ & $33 \pm 12$ & $44 \pm 16$ & $38 \pm 11$ & $29 \pm 21$ & $19 \pm 12$ & $62 \pm 27^{*}$ & $30 \pm 25$ \\
\hline $\mathrm{HAB} / \mathrm{Q} 1(n=18)$ & $42 \pm 18$ & $35 \pm 12$ & $40 \pm 14$ & $38 \pm 9$ & $31 \pm 21$ & $21 \pm 14$ & $55 \pm 30$ & $25 \pm 13$ \\
\hline $\mathrm{HAB} / \mathrm{Q} 2(n=16)$ & $53 \pm 18$ & $39 \pm 17$ & $44 \pm 16$ & $37 \pm 13$ & $24 \pm 13$ & $15 \pm 7$ & $53 \pm 28$ & $28 \pm 27$ \\
\hline $\mathrm{HAB} / \mathrm{H} 1(n=18)$ & $39 \pm 14^{*}$ & $32 \pm 11$ & $42 \pm 12$ & $35 \pm 9$ & $26 \pm 13$ & $20 \pm 13$ & $56 \pm 29$ & $24 \pm 15$ \\
\hline $\mathrm{HAB} / \mathrm{H} 2(n=16)$ & $57 \pm 19 *$ & $42 \pm 17$ & $43 \pm 18$ & $39 \pm 11$ & $29 \pm 23$ & $17 \pm 10$ & $52 \pm 30$ & $32 \pm 28$ \\
\hline
\end{tabular}

Note. $\quad \mathrm{VM}=$ vastus medialis, $\mathrm{VL}=$ vastus lateralis, $\mathrm{BF}=$ biceps femoris, $\mathrm{Gmed}=$ gluteus medius, MVIC = maximal voluntary isometric contraction, $\mathrm{WL}=$ walking lunge, $\mathrm{SSq}=$ split squat, $\mathrm{H} / \mathrm{Q} 1$ = hamstring/quadriceps group $1, \mathrm{H} / \mathrm{Q} 2$ = hamstring/quadriceps group 2, HAB/Q 1 = hip abductor/quadriceps group 1, HAB/Q 2 = hip abductor/quadriceps group 2, $\mathrm{HAB} / \mathrm{H} 1$ = hip abductor/hamstring group 1, HAB/H 2 = hip abductor/hamstring group 2. † significant difference between exercises, *significant difference between related couples of groups.

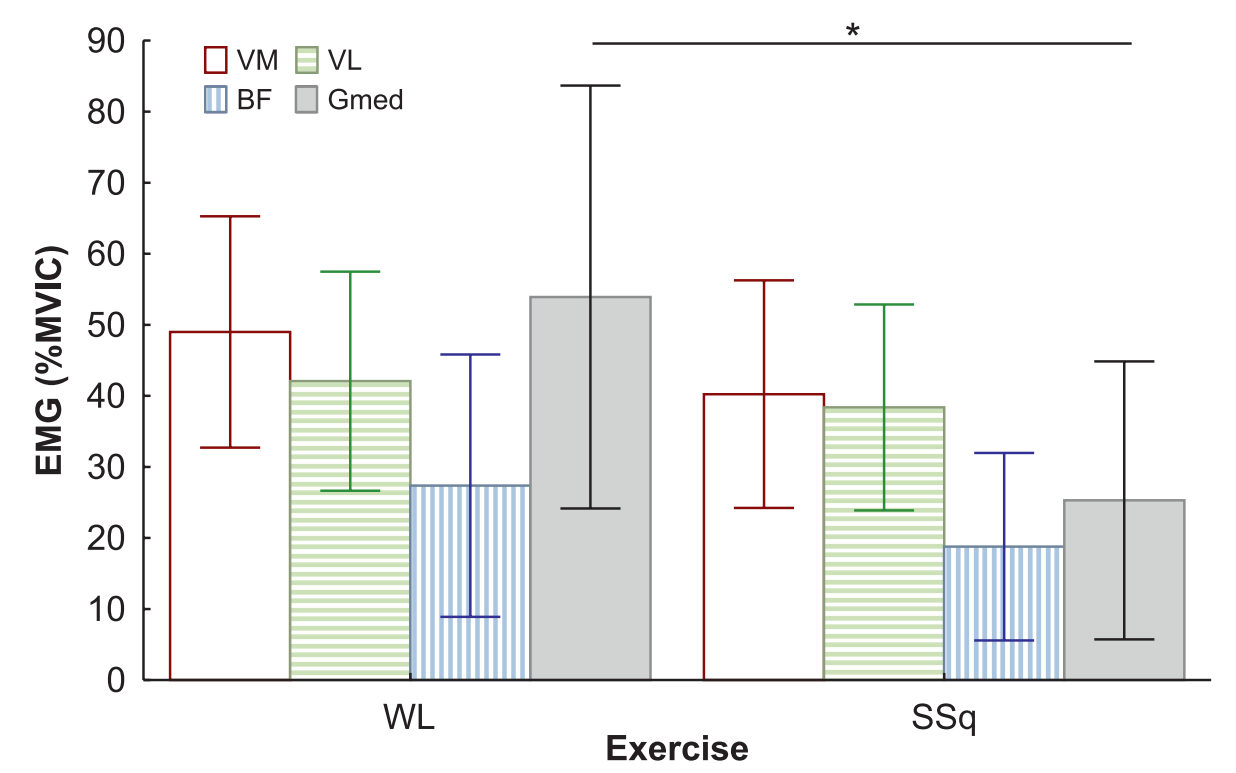

Figure 1. Analysis of variance: differences between exercises. VM = vastus medialis, $\mathrm{VL}=$ vastus lateralis, $\mathrm{BF}=$ biceps femoris, Gmed = gluteus medius, $\mathrm{MVIC}=$ maximal voluntary isometric contraction, $\mathrm{WL}=$ walking lunge, $\mathrm{SSq}=$ split squat, *significant difference between exercises. Rectangular bars represent means, error bars represent standard deviations.

muscle activation during SSqs and WLs, which was found for Gmed, VM and BF, but not for VL. Additionally, there were differences in muscle activation between groups divided by $\mathrm{H} / \mathrm{Q}(0.5)$ or $\mathrm{HAB} / \mathrm{H}$ (1) ratio.

The $\mathrm{H} / \mathrm{Q}$ ratio predicted $\mathrm{VM}$ and $\mathrm{BF}$ amplitude, where the weaker hamstring signifies a lower VM activity and higher BF activity. This finding support the knowledge that lower $\mathrm{H} / \mathrm{Q}$ ratio is associated with knee injury itself (Yusaku, Tomoyuki, Keishoku, Kazuhiko,
\& Eiichi, 2008) and expend it by finding that lower $\mathrm{H} / \mathrm{Q}$ ratio reduce the VM activity. As suggested above, reduced VM activity might cause the knee injury or pathology (Crossley, Bennell, Green, \& McConnell, 2001; Fagan \& Delahunt, 2008). The HAB/Q ratio predicted Gmed and $\mathrm{BF}$ amplitude, where the stronger HAB signify higher activity of Gmed and lower activity of BF. This might be explained by Gmed function in hip and knee stability (Reiman, Bolgla, \& Lorenz, 2009), when appropriate strength of Gmed might decrease the 


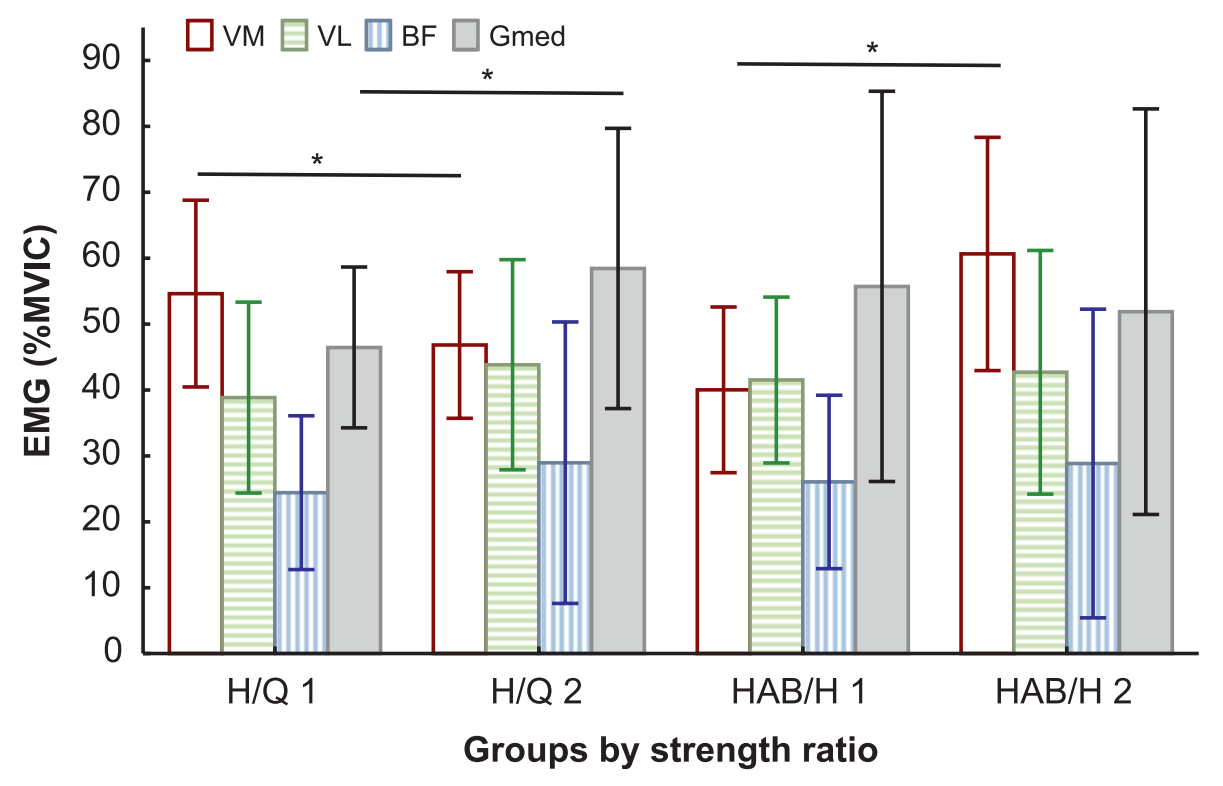

Figure 2. Analysis of variance: differences between groups. VM= vastus medialis, $\mathrm{VL}=$ vastus lateralis, $\mathrm{BF}=$ biceps femoris, $\mathrm{Gmed}=$ gluteus medius, $\mathrm{MVIC}=$ maximal voluntary isometric contraction, H/Q 1 = hamstring/quadriceps group 1, $\mathrm{H} / \mathrm{Q} 2$ = hamstring/quadriceps group 2, HAB/H 1 = hip abductor/hamstring group 1, HAB/H 2 = hip abductor/hamstring group 2. *significant difference between groups. Rectangular bars represent means, error bars represent standard deviations.

demands for BF activity as a quadriceps antagonists. Furthermore $\mathrm{HAB} / \mathrm{H}$ ratio predicted $\mathrm{VM}$ and $\mathrm{Gmed}$ amplitude, where the stronger HAB signify higher Gmed activity and lower VM activity. This might be similar effect to $\mathrm{HAB} / \mathrm{Q}$, where stronger Gmed might decrease the demand for VM activity.

Subjects involved in this study did not have hip abduction, quadriceps or hamstring weakness itself (referenced in Table 1) according to normality data (Bohannon, 1997; Buchanan \& Vardaxis, 2009; Danneskiold-Samsoe et al., 2009; Harbo, Brincks, \& Andersen, 2012; Lehnert, Urban, Procházka, \& Psotta, 2011), so the possibility that higher muscle activity would be find due to its weakness or malfunction was rejected.

The WL showed higher Gmed amplitude than SSq, which might refer to the presence of impact forces during WL. Similar phenomenon was found for Gmed between walking and running, where Gmed peak muscle force during running was higher than during walking (Pandy \& Andriacchi, 2010). Thus it is possible to conclude, that exercise which include the impact forces increase the Gmed activity. The absence of impact forces during SSq might be also the cause of no muscle activation differences between groups divided by muscle strength ratio.

The group with $\mathrm{H} / \mathrm{Q}<0.5$ showed higher $\mathrm{VM}$ activity and lower Gmed activity than group with $\mathrm{H} / \mathrm{Q}>0.5$ during WL. This is in agreement with association between H/Q activity and VM amplitude, where stronger hamstrings signify higher VM amplitude. On the other hand the group with $\mathrm{H} / \mathrm{Q}>0.5$ showed Gmed activity of $62 \%$ MVIC, which is considered a very high activation level (Distefano, Blackburn, Marshall, \& Padua, 2009; French et al., 2010; Reiman, Bolgla, \& Loudon, 2012). The group with $\mathrm{HAB} / \mathrm{H}<1$ showed higher $\mathrm{VM}$ activity than $\mathrm{HAB} / \mathrm{H}<1$, which was in agreement with reciprocal association above. Thus it is possible to conclude that WL exercise is beneficial by strengthening the knee stabilizers like $\mathrm{VM}$ and Gmed for individuals with $\mathrm{H} / \mathrm{Q}<0.5, \mathrm{HAB} / \mathrm{H}<1$ and $\mathrm{H} / \mathrm{Q}>0.5$, respectively. This might be also the reason why WL is considered as traditional rehabilitation exercise (Distefano et al., 2009; Gilleard et al., 1998) that is effective in rehabilitation programs (Alkjær et al., 2002). Furthermore, non-of selected groups showed higher activity of VL in comparison to VM, which means that both SSq and WL does not have negative effect in quadriceps strengthening.

A limitation of this study is the EMG response for selected load of 5RM, which can vary between the individuals due to the genetic profile (Petr et al., 2014) or type of exercise (Čoh \& Žvan, 2011). Although $\mathrm{H} / \mathrm{Q}$ ratio has already been standardized in general population (Danneskiold-Samsøe et al., 2009; Harbo et al., 2012) and athletes (Lehnert et al., 2011; Maly, 
Zahalka, \& Mala, 2014), there are no standards for hip abductor muscles (HAB) to thigh muscle strength. The normative values for HAB strength itself has already been estimated (Buchanan \& Vardaxis, 2009) as well as HAB to hip adduction ratio in athletes (Cichanowski, Schmitt, Johnson, \& Niemuth, 2007; Finnoff et al., 2011; Tyler et al., 2001), but again, without relation to thigh muscles strength. However, by the findings of a relationship between strength ratio and EMG amplitude it is possible to make a recommendation for exercise selection into resistance training programmes.

\section{Conclusions}

The reciprocal ratios of $\mathrm{HAB}, \mathrm{H}$ and $\mathrm{Q}$ are able to predict Gmed, VM and BF activity during WLs and SSq. A higher EMG activity of Gmed was found during WL over the SSq, because WL includes the impact forces as part of lunge movement. WL should be used in resistance training programmes, if the strengthening of Gmed or VM is the aim. WL exercise prefers the activity of VM in individuals with $\mathrm{H} / \mathrm{Q}<0.5$ or $\mathrm{HAB} / \mathrm{H}<1$ and prefers the activity of Gmed in individuals with $\mathrm{H} / \mathrm{Q}>0.5$.

\section{Acknowledgment}

This study was funded by project POST-UP II (No. CZ.1.07/2.3.00/30.0041) co-funded by the European Social Fund and the government of the Czech Republic.

\section{References}

Aagaard, P., Simonsen, E. B., Magnusson, S. P., Larsson, B., \& Dyhre-Poulsen, P. (1998). A new concept for isokinetic hamstring: Quadriceps muscle strength ratio. American Journal of Sports Medicine, 26, 231-237.

Alkjær, T., Simonsen, E. B., Peter Magnusson, S., Aagaard, H., \& Dyhre-Poulsen, P. (2002). Differences in the movement pattern of a forward lunge in two types of anterior cruciate ligament deficient patients: Copers and noncopers. Clinical Biomechanics, 17, 586-593. doi:10.1016/ S0268-0033(02)00098-0

Bennell, K., Duncan, M., Cowan, S., McConnell, J., Hodges, P., \& Crossley, K. (2010). Effects of vastus medialis oblique retraining versus general quadriceps strengthening on vasti onset. Medicine \& Science in Sports \& Exercise, 42, 856-864. doi:10.1249/MSS.0b013e3181c12771

Bohannon, R. W. (1997). Reference values for extremity muscle strength obtained by hand-held dynamometry from adults aged 20 to 79 years. Archives of Physical Medicine and Rehabilitation, 78, 26-32. doi:10.1016/ S0003-9993(97)90005-8
Bolgla, L. A., \& Uhl, T. L. (2005). Electromyographic analysis of hip rehabilitation exercises in a group of healthy subjects. Journal of Orthopedics and Sports Physical Therapy, 35, 487-494.

Bolgla, L. A., \& Uhl, T. L. (2007). Reliability of electromyographic normalization methods for evaluating the hip musculature. Journal of Electromyography and Kinesiology, 17, 102-111. doi:10.1016/j.jelekin.2005.11.007

Boudreau, S. N., Dwyer, M. K., Mattacola, C. G., Lattermann, C., Uhl, T. L., \& McKeon, J. M. (2009). Hip-muscle activation during the lunge, single-leg squat, and step up and over exercises. Journal of Sport Rehabilitation, 18, 91-103.

Brown, L. E., \& Weir, J. (2001). ASEP procedures recommendation I: Accurate assessment of muscular strength and power. Journal of Exercise Physiology Online, 4, 1-21.

Buchanan, P. A., \& Vardaxis, V. G. (2009). Lower-extremity strength profiles and gender-based classification of basketball players ages 9-22 years. Journal of Strength and Conditioning Research, 23, 406-419.

Burnet, E. N., \& Pidcoe, P. E. (2009). Isometric gluteus medius muscle torque and frontal plane pelvic motion during running. Journal of Sports Science and Medicine, 8, 284-288.

Chandler, T. J., \& Brown, L. E. (2008). Conditioning for strength and human performance. Philadelphia, PA: Lippincott Williams \& Wilkins.

Cichanowski, H. R., Schmitt, J. S., Johnson, R. J., \& Niemuth, P. E. (2007). Hip strength in collegiate female athletes with patellofemoral pain. Medicine \& Science in Sports \& Exercise, 39, 1227-1232. doi:10.1249/mss.0b013e3180601109

Čoh, M., \& Žvan, M. (2011). Biodynamic diagnostics of the explosive power of the lower extremities: A case study. Acta Universitatis Carolinae. Kinanthropologica, 47, 16-25.

Crossley, K., Bennell, K., Green, S., \& McConnell, J. (2001). A systematic review of physical interventions for patellofemoral pain syndrome. Clinical Journal of Sport Medicine, $11,103-110$.

Danneskiold-Samsoe, B., Bartels, E. M., Bulow, P. M., Lund, H., Stockmarr, A., Holm, C. C., \& Bliddal, H. (2009). Isokinetic and isometric muscle strength in a healthy population with special reference to age and gender. Acta Physiologica, 197(Suppl. 673), 1-68. doi:10.1111/j.1748-1716.2009.02022.x

Davis, R. B., Õunpuu, S., Tyburski, D., \& Gage, J. R. (1991). A gait analysis data collection and reduction technique. Human Movement Science, 10, 575-587. doi:10.1016/0167-9457(91)90046-Z

Dirnberger, J., Kösters, A., \& Müller, E. (2012). Concentric and eccentric isokinetic knee extension: A reproducibility study using the IsoMed 2000-dynamometer. Isokinetics and Exercise Science, 20, 31-35. doi:10.3233/IES-2012-0437

Distefano, L. J., Blackburn, J. T., Marshall, S. W., \& Padua, D. A. (2009). Gluteal muscle activation during common therapeutic exercises. Journal of Orthopaedic Sports Physical Therapy, 39, 532-540. doi:10.2519/jospt.2009.2796

Dwyer, M. K., Boudreau, S. N., Mattacola, C. G., Uhl, T. L., \& Lattermann, C. (2010). Comparison of lower extremity kinematics and hip muscle activation during rehabilitation tasks between sexes. Journal of Athletic Training, 45, 181-190. 
Fagan, V., \& Delahunt, E. (2008). Patellofemoral pain syndrome: A review on the associated neuromuscular deficits and current treatment options. British Journal of Sports Medicine, 42, 789-795. doi:10.1136/bjsm.2008.046623

Finnoff, J. T., Hall, M. M., Kyle, K., Krause, D. A., Lai, J., \& Smith, J. (2011). Hip strength and knee pain in high school runners: A prospective study. $P M \& R, 3,792-801$. doi:10.1016/j.pmrj.2011.04.007

French, H., Dunleavy, M., \& Cusack, T. (2010). Activation levels of gluteus medius during therapeutic exercise as measured with electromyography: A structured review. Physical Therapy Reviews, 15, 92-105.

Gilleard, W., McConnell, J., \& Parsons, D. (1998). The effect of patellar taping on the onset of vastus medialis obliquus and vastus lateralis muscle activity in persons with patellofemoral pain. Physical Therapy, 78, 25-32.

Harbo, T., Brincks, J., \& Andersen, H. (2012). Maximal isokinetic and isometric muscle strength of major muscle groups related to age, body mass, height, and sex in 178 healthy subjects. European Journal of Applied Physiology and Occupational Physiology, 112, 267-275. doi:10.1007/ s00421-011-1975-3

Holcomb, W. R., Rubley, M. D., Lee, H. J., \& Guadagnoli, M. A. (2007). Effect of hamstring-emphasized resistance training on hamstring:quadriceps strength ratios. Journal of Strength and Conditioning Research, 21, 41-47.

Irish, S. E. J., Millward, A. J., Wride, J., Haas, B. M., \& Shum, G. L. (2010). The effect of closed-kinetic chain exercise and open-kinetic chain exercise on the muscle activity of vastus medialis oblique and vastus lateralis. Journal of Strength and Conditioning Research, 24, 12561262. doi:10.1519/JSC.0b013e3181cf749f

Jakobsen, M. D., Sundstrup, E., Andersen, C. H., Aagaard, P., \& Andersen, L. L. (2013). Muscle activity during leg strengthening exercise using free weights and elastic resistance: Effects of ballistic vs. controlled contractions. Human Movement Science, 32, 65-78. doi:10.1016/j. humov.2012.07.002

Kivlan, B. R., \& Martin, R. L. (2012). Functional performance testing of the hip in athletes: A systematic review for reliability and validity. International Journal of Sports Physical Therapy, 7, 402-412.

Kong, P. W., \& Burns, S. F. (2010). Bilateral difference in hamstrings to quadriceps ratio in healthy males and females. Physical Therapy in Sport, 11, 12-17. doi: $10.1016 / j . p t s p .2009 .09 .004$

Larson-Hall, J. (2009). A guide to doing statistics in second language research using SPSS. New York, NY: Routledge.

Leetun, D. T., Ireland, M. L., Willson, J. D., Ballantyne, B. T., \& Davis, I. M. (2004). Core stability measures as risk factors for lower extremity injury in athletes. Medicine \& Science in Sports \& Exercise, 36, 926-934.

Lehnert, M., Urban, J., Procházka, J. H., \& Psotta, R. (2011). Isokinetic strength of knee flexors and extensors of adolescent soccer players and its changes based on movement speed and age. Acta Universitatis Palackianae Olomucensis. Gymnica, 41(2), 45-53.

Maly, T., Zahalka, F., \& Mala, L. (2014). Muscular strength and strength asymmetries in elite and sub-elite professional soccer players. Sport Science, 7, 26-33.
O’Sullivan, K., Smith, S. M., \& Sainsbury, D. (2010). Electromyographic analysis of the three subdivisions of gluteus medius during weight-bearing exercises. Sports Medicine, Arthroscopy, Rehabilitation, Therapy \& Technology, 2, Article ID 17. doi:10.1186/1758-2555-2-17

Pandy, M. G., \& Andriacchi, T. P. (2010). Muscle and joint function in human locomotion. Annual Review of Biomedical Engineering, 12, 401-433. doi:10.1146/ annurev-bioeng-070909-105259

Petr, M., Št'astný, P., Pecha, O., Šteffl, M., Šeda, O., \& Kohlíková, E. (2014). PPARA intron polymorphism associated with power performance in 30-s anaerobic Wingate Test. PLoS ONE, 9, Article ID e107171. doi:10.1371/journal.pone.0107171

Powers, C. M., Landel, R., \& Perry, J. (1996). Timing and intensity of vastus muscle activity during functional activities in subjects with and without patellofemoral pain. Physical Therapy, 76, 946-955.

Reiman, M. P., Bolgla, L. A., \& Lorenz, D. (2009). Hip function's influence on knee dysfunction: A proximal link to a distal problem. Journal of Sport Rehabilitation, 18, 33-46.

Reiman, M. P., Bolgla, L. A., \& Loudon, J. K. (2012). A literature review of studies evaluating gluteus maximus and gluteus medius activation during rehabilitation exercises. Physiotherapy in Theory and Practice, 28, 257-268. doi:10. 3109/09593985.2011.604981

Segal, N. A., Glass, N. A., Torner, J., Yang, M., Felson, D. T., Sharma, L., ... Lewis, C. E. (2010). Quadriceps weakness predicts risk for knee joint space narrowing in women in the MOST cohort. Osteoarthritis and Cartilage, 18, 769775. doi:10.1016/j.joca.2010.02.002

Sheskin, D. J. (2003). Handbook of parametric and nonparametric statistical procedures. Boca Raton, FL: CRC Press.

Stastny, P., Lehnert, M., Zaatar, A., Svoboda, Z., Xaverova, Z., \& Jelen, K. (2014). Knee joint muscles neuromuscular activity during load-carrying walking. Neuroendocrinology Letters, 35, 633-639.

Stastny, P., Lehnert, M., Zaatar, A., Svoboda, Z., Xaverova, Z., \& Pietraszewski, P. (2015). The gluteus medius vs. thigh muscles strength ratio and their relation to electromyography amplitude during a farmer's walk exercise. Journal of Human Kinetics, 45, 157-165. doi:10.1515/ hukin-2015-0016

Tyler, T. F., Nicholas, S. J., Campbell, R. J., \& McHugh, M. P. (2001). The association of hip strength and flexibility with the incidence of adductor muscle strains in professional ice hockey players. The American Journal of Sports Medicine, 29, 124-128.

Windolf, M., Götzen, N., \& Morlock, M. (2008). Systematic accuracy and precision analysis of video motion capturing systems-exemplified on the Vicon-460 system. Journal of Biomechanics, 41, 2776-2780. doi:10.1016/j. jbiomech.2008.06.024

Yusaku, S., Tomoyuki, S., Keishoku, S., Kazuhiko, S., \& Eiichi, S. (2008). Strength deficits identified with concentric action of the hip extensors and eccentric action of the hamstrings predispose to hamstring injury in elite sprinters. Journal of Orthopaedic and Sports Physical Therapy, 38, 457-464. doi:10.2519/jospt.2008.2575 